doi: $10.13108 / 2021-13-2-8$

\title{
ON GENERALIZATIONS OF CHEBYSHEV POLYNOMIALS AND CATALAN NUMBERS
}

\author{
B.S. BYCHKOV, G.B. SHABAT
}

\begin{abstract}
We provide possible directions of generalizations of earlier found relations between the Chebyshev polynomials and the Catalan numbers arising in studying commuting difference operators. These generalizations are mostly related with ideas proposed by N.H. Abel in his publication in 1826, which then were reproduced by many authors in a modern language. As generalization of Chebyshev polynomials, we propose to consider polynomials with exactly two critical values well-studied in a so-called theory of dessins d'enfants. The Catalan numbers are located in the first column of the table of HarerZagier numbers related with the distribution by genus of orientable sewing of polygons with even number of sides. The commuting difference operators are implicitly contained in the Abel theory, who studied quasi-elliptic integrals, namely, the elliptic integrals of 3rd kind integrable in terms of logarithms. In the present work we formulate conjectures on relation between the main Abel theorem and commuting semi-infinite matrices. In the work we provide calculations supporting the conjectured relations.
\end{abstract}

Keywords: Chebyshev polynomials, Catalan numbers, Harer-Zagier numbers, polynomial Pell equation, dessins d'enfants.

Mathematics Subject Classification: 39A70, 33C75

\section{INTRODUCTION}

A.B. Shabat, regarding himself primarily as a specialist in the theory of differential equations, had a broad mathematical outlook. He was able to see unexpected connections between branches of mathematics that were far from each other, but he preferred to fix his ideas not by developing general theories, but by considering specific examples.

His last lifetime publication was paper [7] published jointly with one of the co-authors of this paper, in which relations were established between commuting semi-infinite matrices, Chebyshev polynomials, and Catalan numbers. We have no doubt that these connections admit various generalizations, and we are sure that if A.B. Shabat lived longer, he would have found these generalizations.

In this paper, we outline one of the directions of such generalizations.

\section{Chebyshev polynomials and Catalan numbers}

We recall brielfy the mentioned relation from paper [7].

B.S. Bychkov, G.B. Shabat, On generalizations of Chebyshev polynomials and Catalan numBERS.

(C) Bychkov B.S., Shabat G.B. 2021.

The work is supported by the grant of Theoretical Physics and Mathematics Advancement Foundation "BASIS". The research by G.B. Shabat is supported by Simons foundation.

Submitted April 21, 2021. 
Chebyshev polynomials ${ }^{1}$ are defined in many ways and we provide just two of them: a closed formula

$$
\mathrm{T}_{n}(u)=\cos (n \arccos u)
$$

and a recurrent relation

$$
\mathrm{T}_{n+1}(u)=2 u \mathrm{~T}_{n}(u)-\mathrm{T}_{n-1}(u)
$$

with initial conditions $\mathrm{T}_{0}(u)=1$ and $\mathrm{T}_{1}(u)=u$; see, for instance, [8].

Catalan numbers are also determined in many ways, see, for instance, 11.

We omit several combinatorial interpretations of Catalan numbers; unfortunately, the combinatorics is absent in the present paper. We defined the Catalan numbers $c_{0}, c_{1}, \ldots$ by means of a generating function

$$
\sum_{n=0}^{\infty} c_{n} u^{n}=\frac{1-\sqrt{1-4 u}}{2 u} .
$$

A short formulation of one of the main results of paper [7] is that quotients of neighbouring Chebyshev polynpomials are close to a truncated generating function of the Catalan numbers. For instance,

$$
\begin{aligned}
\frac{\mathrm{T}_{8}\left(\frac{1}{2 M}\right)}{\mathrm{T}_{7}\left(\frac{1}{2 M}\right)} & =\frac{1}{M}-M-M^{3}-2 M^{5}-5 M^{7}-14 M^{9}-42 M^{11}-\ldots \\
& =\frac{1}{M}-c_{0} M-c_{1} M^{3}-c_{2} M^{5}-c_{3} M^{7}-c_{4} M^{9}-4 c_{5} M^{11}-\ldots
\end{aligned}
$$

At present we do not know direct generalizations of these identities but we conjecture that they are possible. However, the objects in the left hand side and the right hand side of identities (2.4) admit natural generalizations and we will discuss them in the next sections. Here we mention a theory, in the framework of which we hope to find more conceptual explanations of the matter.

A.B. Shabat seemed to feel a general mathematical perspective explaining the nature of the discussed relations. And our task is to restore it soon or later.

\section{Generalized Chebyshev polynomials}

One of the properties of usual Chebyshev polynomials $\mathrm{T}_{n}$ is that they have only two finite critical values; this property is obvious if we use definition (2.1). This is a base for one of the generalizations we have in mind.

An arbitrary polynomial $P \in \mathbb{C}[u]$ of order at least two is called a generalized Chebyshev polynomia $\left.\right|^{2}$ if there exist numbers $a, b \in \mathbb{C}$ such that

$$
\left[P^{\prime}(u)=0\right] \Longrightarrow[P(u) \in\{a, b\}] .
$$

It is easy to show that in this case the pre-image $P^{-10}[a, b]$ of the segment connecting critical points is a tree in the complex plane $\mathbb{C}$. Moreover, this procedure of associating a planar tree with a generalized Chebyshev polynomial is a one-to-one correspondence between the similarity classes of Chebyshev generalized polynomials (that is, the orbit of the action of the square of the group $\mathbb{C}^{\times} \times \mathbb{C}$ on the polynomials by the linear changes in the argument and in the image) and isotopic classes of planar tress; see [6].

This correspondence gives an unbounded amount of generalized Chebyshev polynomials although it is a nontrivial computing problem to find a particular polynomial by a corresponding planar tree. Usual Chebyshev polynomials correspond to chains.

\footnotetext{
${ }^{1}$ of the first kind, and we do not mention this below.

${ }^{2}$ or Shabat polynomial and we mean here not A.B. Shabat but one of the co-authors of the present paper.
} 
We shall need just one property of generalized Chebyshev polynomials.

Let $P$ be a generalized Chebyshev polynomial with a leading coefficient 1 and with critical values $\{a, b\}=\{ \pm 1\}$; in each similarity class we can choose such element. The vertices of the tree corresponding to the polynomial $P$ are pre-images of critical values \pm 1 and they split into disjoint union

$$
V_{P}=V_{P}^{+} \coprod V_{P}^{-}
$$

where

$$
V_{P}^{ \pm}=\{u \in \mathbb{C} \mid P(u)= \pm 1\} .
$$

Then the following factorization holds:

$$
P \pm 1=\prod_{\alpha \in V_{P}^{ \pm}}(u-\alpha)^{v_{\alpha}},
$$

where $v_{\alpha}$ is a valence of a vertex $\alpha$ in the tree corresponding to a polynomial $P$.

We also define

$$
P_{\text {odd }}:=\prod_{\alpha \in V_{P}: v_{\alpha} \in 2 \mathbb{N}+1}(u-\alpha)
$$

a polynomial, the simple roots of which are vertices of odd valence of the tree associated with the polynomial $P$.

We note that $\operatorname{deg} P_{\text {odd }} \geq 2$ : each tree has at least two vertices of odd valence and there are exactly two such vertices if and only if the tree is a chain and then the corresponding polynomial is a usual Chebyshev polynomial.

Theorem 3.1. Let $P$ be a generalized Chebyshev polynomial. Then there exist polynomials $X, Y \in \mathbb{C}[u]$ such that

$$
X^{2}-P_{\text {odd }} Y^{2}=1 \text {. }
$$

Proof. Multiplying identities (3.3), we obtain

$$
P^{2}-1=\prod_{\alpha \in V_{P}}(u-\alpha)^{v_{\alpha}} .
$$

In order to obtain (3.4) it remains to let $X:=P$ and to select a full square in the right hand side.

The statement of the proven theorem means that each generalized Chebyshev polynomial gives a coefficient of a Pell's polynomial equation having nontrivial solutions.

\section{QUASI-ELLIPTIC INTEGRALS}

In paper [1] N.H. Abel considered an issue on which integrals of form

$$
\int \frac{\rho \mathrm{d} u}{\sqrt{R}}
$$

can be expressed in terms of elementary functions; here $\rho$ and $R$ are polynomials over $\mathbb{C}$. More precisely, he studied for which polynomials $R$ of an even degree there exists a polynomial $\rho$ with the mentioned property. In view of obvious reasons, here it is sufficient to consider only polynomials with no multiple roots.

The polynomials $R$ satisfying the discussed conditions will be called abelian.

In paper [1], there were provided the following properties of the polynomials $R$ equivalent to the abelian property. 
(1) There exist polynomials $X$ and $Y$ of positive degrees satisfying the polynomial Pell's equation

$$
X^{2}-R Y^{2}=1 .
$$

(2) The coefficients $a_{0}, a_{1}, \cdots \in \mathbb{C}[u]$ of the expansion into a continued fraction

$$
\sqrt{R}=a_{0}+\frac{1}{a_{1}+\frac{1}{a_{2}+\frac{1}{a_{3}+\cdots}}}
$$

are periodic.

At a modern mathematical language, condition (1) can be equivalently reformulated as follows. We consider a hyperelliptic curve, a smooth affine model $\ddot{\mathbf{X}}_{R}$ of which is defined by the equation

$$
v^{2}=R(u)
$$

This model is of the form

$$
\ddot{\mathbf{X}}_{R}=\mathbf{X}_{R} \backslash\left\{\underline{\infty}_{ \pm}\right\}
$$

where $\mathbf{X}_{R}$ is a smooth projective model; if $R=u^{2 g+2}+\ldots$, then at punctured neighbourhoods of the points $\underline{\infty}_{ \pm}$an asymptotic identity $v \approx \pm u^{g+1}$ holds true. An equivalent to (1) condition is

$(1)^{\prime} . \underline{\infty}_{+}-\underline{\infty}_{-} \in \operatorname{tors}(\operatorname{Pic} \mathbf{X})$, the difference of infinite points has a finite order.

Usual Chebyshev polynomials correspond to a "degenerate" case of the curve $\mathbf{X}$ of genus zero, the abelian polynomial $R=u^{2}-1$, standard integrals $\int \frac{\rho \mathrm{d} u}{\sqrt{u^{2}-1}}$, solutions (for each natural $n$ ) of the polynomial Pell's equation of form $X=\mathrm{T}_{n}, Y=\mathrm{U}_{n-1}$ (Chebyshev polynomials of second kind) and the expansion into continued fraction

$$
\begin{aligned}
\sqrt{u^{2}-1} & =u+\left(\sqrt{u^{2}-1}-u\right)=u+\frac{1}{u+\sqrt{u^{2}-1}} \\
& =u+\frac{1}{2 u+\left(\sqrt{u^{2}-1}-u\right)}=u+\frac{1}{2 u+\frac{1}{2 u+\frac{1}{2 u+\ldots}}} .
\end{aligned}
$$

Let us provide a non-trivial example of a curve of genus 1 . It is defined by the equation

$$
v^{2}=u^{4}+u^{3}+\frac{1}{4} u^{2}+2 u+1=: R
$$

and a pair of solutions to Abel-Pell equation $X^{2}-R y^{2}=1$ are the polynomials

$$
X=u^{3}+\frac{1}{2} u^{2}+1, \quad Y=u .
$$

The expansion into the continued fraction is of a form

$$
\sqrt{u^{4}+u^{3}+\frac{1}{4} u^{2}+2 u+1}=u^{2}+\frac{u}{2}+\frac{2}{2 u+\frac{1}{u^{2}+\frac{u}{2}+\frac{1}{2 u+\frac{1}{u^{2}+\frac{u}{2}+\ldots}}}},
$$

and the quasi-elliptic integral reads as

$$
\int \frac{(6 u+2) \mathrm{d} u}{\sqrt{u^{4}+u^{3}+\frac{1}{4} u^{2}+2 u+1}}=\log \frac{u^{3}+\frac{1}{2} u^{2}+1+u \sqrt{u^{4}+u^{3}+\frac{1}{4} u^{2}+2 u+1}}{u^{3}+\frac{1}{2} u^{2}+1-u \sqrt{u^{4}+u^{3}+\frac{1}{4} u^{2}+2 u+1}} .
$$


In the 19th century, such integrals were highly valued and finding one was sufficient for publication. It follows from the results of the previous section that each planar tree allows one to construct a quasi-elliptic integral.

We believe that generalized Chebyshev polynomials are a natural candidate for generalizations of the results of [7]. The corresponding continued fractions are interpreted in terms of difference second order operators, see, for instance, [10], also present in [7].

\section{HARER-ZAGIER NUMBERS}

These numbers $\varepsilon_{g}(n)$ were introduced in paper [2]. Each such number is defined as the number of gluing of a $(2 n)$-gon into an orientable surface of genus $g$. They satisfy various recurrent relations being valid due to the generating function

$$
\left(\frac{1+x}{1-x}\right)^{y}=: 1+2 x y+2 \sum_{n=1}^{\infty} \sum_{g=0}^{\left\lfloor\frac{n+1}{2}\right\rfloor} \frac{\varepsilon_{g}(n)}{(2 n-1) ! !} x^{n+1} y^{n+1-2 g}
$$

we note that the coefficient $\frac{\varepsilon_{g}(n)}{(2 n-1) ! !}$ can be interpreted as the probability of the event that a random oriented gluing of a $(2 n)$-gon is of genus $g$, see, for instance, [5].

Catalan numbers form a zero column of Harer-Zagier table

$$
c_{n} \equiv \varepsilon_{0}(n) \text {. }
$$

Other columns behave in a similar way. For instance, the column of genus 1 satisfies the recurrent relation

$$
\varepsilon_{1, n+1}=\frac{4 n+2}{n-1} \varepsilon_{1, n}
$$

close to the relation

$$
c_{n+1}=\frac{4 n+2}{n+2} c_{n}
$$

for the Catalan number. The generating function

$$
\sum_{n=1}^{\infty} \varepsilon_{1, n} u^{n}=\frac{1}{(1-4 x)^{\frac{5}{2}}}
$$

is also close to Definition (2.3).

The mentioned parallels show that the Harer-Zagier numbers are natural candidates for generalizations of the results in [7]. Of course, we would like to find these generalizations not only formally, but also at a conceptual level; such possibility is supported by the role of these numbers in [2].

\section{On COMMUting DifFERENCE OPERATORS}

Another main result of paper [7] is a natural connection between the Chebyshev polynomials and the elements of some commuting difference operators. Namely, we consider the problem on commuting a tridiagonal matrix $A$ with the Vandermonde matrix $\Lambda$

$$
A=\left(\begin{array}{cccccc}
a_{1} & b_{1} & 0 & \ldots & & \\
b_{1} & a_{2} & b_{2} & 0 & \ldots & \\
\ldots & \ldots & \ldots & \ldots & \ldots & \\
0 & \ldots & b_{n-1} & a_{n} & b_{n} & 0 \ldots \\
\ldots & \ldots & \ldots & \ldots & \ldots &
\end{array}\right), \quad \Lambda=\left(\begin{array}{cccccc}
1 & 1 & 1 & \ldots & 1 & \\
1 & \lambda & \lambda^{2} & \ldots & \lambda^{n} & \ldots \\
\ldots & \ldots & \ldots & \ldots & \ldots & \\
1 & \lambda^{n} & \lambda^{2 n} & \ldots & \lambda^{n^{2}} & \ldots \\
\ldots & \ldots & \ldots & \ldots & \ldots &
\end{array}\right) .
$$


Here $\lambda \in \mathbb{C}$ is a free parameter and a semi-infinite matrix $\Lambda$ is regarded as given. The entries of the sought tridiagonal matrix $A$ are supposed to be rational functions $\lambda$ satisfying the "realness" condition.

It turns out that under appropriate initial conditions $a_{1}=1, b_{1}=0$, the matrices $A$ and $\Lambda$ commute if and only if the entries of the matrix $A$ are rational functions in $\lambda$ with poles at the points 0 and -1 and the functions $\varphi_{n}(\lambda)=1-a_{n+1}(\lambda)$ satisfy a recurrent relations, cf. (2.2):

$$
\varphi_{n+1}+\varphi_{n-1}=\mu \varphi_{n}+2, \quad \varphi_{n}=\frac{1}{\lambda^{n-1}}\left(\frac{\lambda^{n}-1}{\lambda-1}\right)^{2}=1-a_{n+1}, \quad n \geq 0
$$

where $\mu=\lambda+\frac{1}{\lambda}$.

A classical work by Van Moerbeke and Mumford [4] referring to [9] paper by Zakharov and Shabat relates many ideas and constructions from various fields in mathematics. Among other things, it points to a close parallel between the commutative rings of difference and differential operators; in both cases, in the 70s of the last century, the connection between algebra (the structure of these rings), geometry (dynamics of straight motion along the Jacobians of the spectra of these rings) and differential equations (explicit solutions of these equations in theta functions) was clarified. The very subject of this work indicates the intersection with [7]; for example, tridiagonal (semi)infinite matrices appear everywhere, among which commuting ones are distinguished.

The geometric theory of commuting difference operators developed intensively in the following decades in several interesting directions, see, for example, [3].

We however note that Abel's theory, published in 1826, is not always cited in these works and, perhaps, some connections have yet to be realized.

\section{Conclusion}

We very briefly outlined some directions for the developing the ideas by A.B. Shabat, developed by him in the last years of his life and work (for him, these concepts coincided). As people close to him, we see our task in keeping these ideas not being forgotten, but, on the contrary, being clarified, refined and deepened; we hope to contribute as much as possible.

We will be happy if this paper will stimulate some of our colleagues, friends and students of A.B. Shabat or new people, to participate in this work.

\section{ACKNOWLEDGMENTS}

The authors are thankful to S.V. Smirnov for useful discussions.

\section{BIBLIOGRAPHY}

1. N.H. Abel. Sur l'integration de la formule differentielle $\frac{\rho \mathrm{d} z}{\sqrt{R}}, R$ et $\rho$ etant des fonctions entieres // J. Reine Angew. Math. 1, 185-221 (1826).

2. J. Harer, D. Zagier. The Euler characteristic of the moduli space of curves // Invent. Math. 85, 457-485 (1986).

3. A. Izosimov. Pentagrams, inscribed polygons, and Prym varieties // El. Res. An. in Math. Sc. 23, 25-40 (2016).

4. P. Van Moerbeke, D. Mumford. The spectrum of difference operators and algebraic curves // Acta Math. 143, 93-154 (1979).

5. B.G. Pittel. Another Proof of the Harer-Zagier Formula // Electron. J. Comb. 23:1 (2016).

6. G. Shabat, A. Zvonkin. Plane trees and algebraic numbers // Contemp. Math. 178, 233-275 (1994). 
7. A.E. Artisevich, B.S. Bychkov, A.B. Shabat. Chebyshev polynomials, Catalan numbers, and tridiagonal matrices // Teor. Matem. Fiz. 204:1, 3-9 (2020). [Theor. Math. Phys. 204:1, 837$842(2020)$.]

8. N. Vasiliev, A. Zelevinskii. Chebyshev polynomials and recurrent relations // Kvant. 1, 12-19 (1982). (in Russian).

9. V.E. Zakharov, A.B. Shabat. A scheme for integrating the nonlinear equations of mathematical physics by the method of the inverse scattering problem. I. // Funkts. Anal. Pril. 8:3, 54-56 (1974). [Funct. Anal. Appl. 8:3, 226-235 (1974).]

10. A.Ya. Khinchin. Continued fractions. Fizmatlit, Moscow (1960). [University of Chicago Press, Chicago (1964).]

11. G.B. Shabat. Several points of views on Catalan numbers // in "Elements of mathematics", eds. A.A. Zaslavsky, A.B. Skopenkov, M.B. Skopenkov. MCCME, Moscow (2018). (in Russian).

Boris Sergeevich Bychkov,

HSE University,

Usacheva str. 6,

119048, Moscow, Russia

P.G. Demidov Yaroslavl State University,

Sovetskaya str. 14,

150003, Yaroslavl, Russia

E-mail: bbychkov@hse.ru

Georgii Borisovich Shabat,

Russian State University for The Humanities,

Miusskaya sq. 6,

125993, Moscow, Russia

E-mail: george.shabat@gmail.com 